\title{
Third-order explicit two-step Runge-Kutta-Nyström method for solving second-order ordinary differential equations
}

\begin{abstract}
A two-stage explicit two-step Runge-Kutta-Nyström (TSRKN) method is constructed for the numerical integration of special second-order IVPs. Algebraic order conditions of the method are obtained and third-order method is derived. A standard set of test problems are tested and comparisons on the numerical results are made with existing Runge-Kutta-Nyström (RKN) and Runge-Kutta (RK) methods of the same order using constant step size. The numerical results show's its clear advantage in terms of function evaluation.
\end{abstract}

Keyword: Two-step Runge-Kutta-Nystrom method; Order conditions; Second-order ordinary differential equations; Constant step size 